\title{
Nucleons, nuclear matter and quark matter: a unified NJL approach
}

\author{
S Lawley ${ }^{1,2}$, W Bentz ${ }^{3}$ and A W Thomas ${ }^{2}$ \\ ${ }^{1}$ Special Research Centre for the Subatomic Structure of Matter, University of Adelaide, \\ Adelaide SA 5005, Australia \\ 2 Jefferson Lab, 12000 Jefferson Avenue, Newport News, VA 23606, USA \\ ${ }^{3}$ Department of Physics, School of Science, Tokai University Hiratsuka-shi, \\ Kanagawa 259-1292, Japan \\ E-mail: slawley@jlab.org
}

Received 13 February 2006

Published 7 April 2006

Online at stacks.iop.org/JPhysG/32/667

\begin{abstract}
We use an effective quark model to describe both hadronic matter and deconfined quark matter. By calculating the equations of state and the corresponding neutron star properties, we show that the internal properties of the nucleon have important implications for the properties of these systems.
\end{abstract}

(Some figures in this article are in colour only in the electronic version)

\section{Introduction}

Several recent studies have shown the advantages of using composite nucleons in finite density calculations [1-3]. For example, in some models the saturation mechanism of the system can be related to how the structure of the nucleon changes with density [4]. While saturating nuclear systems can also be readily obtained using point-like nucleons [5], if one wishes to explore the transition to quark matter it is desirable to start with a description of the nucleon on the quark level. Although the nucleon is a complex object, some simplification arises by treating it as an interacting quark-diquark state in the Faddeev approach. It turns out that this picture of the nucleon has considerable appeal, both theoretical and observational ${ }^{4}$. For example, it nicely reproduces the light baryon spectrum [7], while a calculation without diquark correlations predicts an abundance of missing resonances [8]. Indeed, the widespread consensus that quark pairing is favourable in high density quark matter [9], where perturbative QCD has some predictive power, lends support to the idea that diquark correlations within the nucleon are important too [6].

In this work, we consider the possibility of a connection between the diquark correlations within the nucleon and the pairing of quarks anticipated in colour superconducting quark

4 For a discussion on the evidence in favour of diquarks see [6]. 
matter. We work with the assumption of two flavours. At zero temperature and normal nuclear matter density strange quarks certainly do not feature in the dynamics of the system, so this is a reasonable starting point. At higher densities strangeness could play a role through the formation of hyperons, kaon condensates and/or strange quark matter, depending on which of these phase transitions is favoured and in what sequence they appear with respect to density [10-13]..$^{5}$ Actually, there are numerous possibilities for quark pairing and condensation in high density matter, especially if strangeness is introduced. Although these ideas can all be investigated within the framework we are using, we wish to concentrate here on the question of whether a unified description of diquark interactions can be achieved for both the nuclear matter (NM) and quark matter (QM) phases. For this purpose, we consider the simplest possibility that two-flavour hadronic matter goes directly to two-flavour QM, using the Nambu-JonaLasinio (NJL) model to describe both phases. In NJL type models, it has been shown that the mass of the strange quark tends to inhibit the formation of strange QM in the density region relevant to compact stars [15-18]. The possibility of pseudoscalar condensation is considered in [19-23], and the competition between the pseudoscalar condensates and the two-flavour superconducting phase is examined in [24].

The NJL model was originally developed to describe interactions between structureless nucleons [25]. It has been shown that this approach does not lead to nuclear stability [26-28]. The idea of the NJL model in its present form is to describe QCD at low energies by assuming point-like interactions between quarks. This approximation is justified by the fact that gluon degrees of freedom should be of relatively minor importance at low energies. Therefore, it is sufficient to construct a model where the gluon interactions are 'frozen in', meaning they are only present implicitly through the couplings of the model. The general form of the NJL Lagrangian density is given by

$$
\mathcal{L}_{\mathrm{NJL}}=\bar{\psi}(\mathrm{i} \not \partial-m) \psi+\sum_{\alpha} G_{\alpha}\left(\bar{\psi} \Gamma_{\alpha} \psi\right)^{2}
$$

where $\bar{\psi}$ and $\psi$ are the quark fields, $m$ is the current quark mass and $G_{\alpha}$ are the coupling constants associated with the various interaction channels. The interaction Lagrangian density can be expressed in a variety of equivalent forms using Fierz transformations [29]. Here, we decompose it into $\bar{q} q$ and $q q$ interaction terms as follows:

$$
\begin{aligned}
\mathcal{L}_{\mathrm{NJL}}^{\text {int }}= & G_{\pi}(\bar{\psi} \psi)^{2}-G_{\pi}\left(\bar{\psi} \gamma_{5} \tau \psi\right)^{2} \\
& -G_{\omega}\left(\bar{\psi} \gamma^{\mu} \psi\right)^{2}-G_{\rho}\left(\bar{\psi} \gamma^{\mu} \tau \psi\right)^{2} \\
& +G_{s}\left(\bar{\psi} \gamma_{5} C \tau_{2} \beta^{A} \bar{\psi}^{T}\right)\left(\psi^{T} C^{-1} \gamma_{5} \tau_{2} \beta^{A} \psi\right) \\
& +G_{a}\left(\bar{\psi} \gamma_{\mu} C \tau \tau_{2} \beta^{A} \bar{\psi}^{T}\right)\left(\psi^{T} C^{-1} \gamma_{\mu} \tau_{2} \tau \beta^{A} \psi\right) .
\end{aligned}
$$

In order of appearance the above terms correspond to the scalar, pseudoscalar, vector-isoscalar and vector-isovector $\bar{q} q$ interactions, and the last two terms correspond to the scalar and the axial vector diquark channels. The colour matrices are given by $\beta^{A}=\sqrt{3 / 2} \lambda^{A}(A=2,5,7)$, indicating that these are attractive, colour anti-symmetric diquark channels. In the NM phase the diquark interactions will lead to colour singlet nucleons, and in the QM phase to colour superconducting pairs. Indeed, QCD supports the idea that there is a strong attraction in the colour anti-symmetric flavour singlet scalar channel, leading to the formation of condensed scalar diquarks (the so-called 2SC phase) [6]. In our model, these condensed pairs should

5 The possibility of three-flavour quark stars was first investigated in 1970 by Itoh [14]. 
arise from the same interaction $(1.2 c)$ as the scalar diquarks in the nucleon constructed in the Faddeev approach ${ }^{6}$.

Because the NJL model is non-renormalizable, the model is only fully specified after the choice of regularization scheme [30]. In our work, quark confinement is ensured mathematically through the introduction of a finite infrared cut-off $\left(\Lambda_{\mathrm{IR}}\right)$ in the proper time regularization scheme [31, 32]. In this method, the unphysical quark decay thresholds are eliminated [1], as also found in the Dyson-Schwinger approach [32]. Of course, in the deconfined phase the infrared cut-off will be set to zero [33].

\section{Nucleons and nuclear matter}

To describe NM, we first consider the nucleon and its internal degrees of freedom. Incorporating the quark substructure of the nucleon through the Faddeev approach will allow us to examine how the nucleon properties change with density. The homogeneous Faddeev equation for the vertex function $\Gamma_{N}$ in the nucleon channel $(J, T)=(1 / 2,1 / 2)$ has the form $\Gamma_{N}=Z \Pi_{N} \Gamma_{N}$, where $Z$ is the quark exchange kernel and $\Pi_{N}$ the product of the quark and diquark propagators [29]. For our present finite density calculations we restrict ourselves to the static approximation [34], where the momentum dependence of the quark exchange kernel $Z$ is neglected and a static parameter $c$ is instead introduced to reproduce the main features of the exact Faddeev calculation [1]. Then, $\Pi_{N}$ becomes effectively the quark-diquark bubble graph given by

$$
\begin{aligned}
\Pi_{N}^{a b}(p) & =\int_{\mathrm{PT}} \frac{\mathrm{d}^{4} k}{(2 \pi)^{4}} \tau^{a b}(p-k) S(k) \\
& =\int_{\mathrm{PT}} \frac{\mathrm{d}^{4} k}{(2 \pi)^{4}}\left(\begin{array}{cc}
\tau_{s}(p-k) & 0 \\
0 & \tau_{a}^{\mu \nu}(p-k)
\end{array}\right) S(k),
\end{aligned}
$$

where $\tau_{s}$ and $\tau_{a}^{\mu \nu}$ refer to the scalar and axial vector components of the diquark $t$-matrices, respectively ${ }^{7}$, and $S(k)$ is the constituent quark propagator. The subscript (PT) on the integral refers to the fact that we use the proper time regularization scheme [37], which involves an infrared $\left(\Lambda_{\mathrm{IR}}\right)$ and an ultraviolet $\left(\Lambda_{\mathrm{UV}}\right)$ cut-off as explained in $[31,32]$. The nucleon mass follows from the requirement that the Faddeev kernel $K \equiv Z \Pi_{N}$ has eigenvalue 1. In a similar way, the mass of the Delta resonance can be calculated from the pole position in the $(J, T)=(3 / 2,3 / 2)$ channel, where only the axial vector diquark contributes.

Here, we wish to use a more accurate description of the nucleon and delta masses, including also the pion cloud contributions [38]. This will enable us to consider a quantitative comparison of the scalar pairing found in the confined and deconfined phases. We take into consideration the self-energy contributions corresponding to the $N \rightarrow N \pi \rightarrow N$ and $N \rightarrow \Delta \pi \rightarrow N$ processes for the nucleon and the $\Delta \rightarrow \Delta \pi \rightarrow \Delta$ and $\Delta \rightarrow N \pi \rightarrow \Delta$ processes for $\Delta$ [38]. The corresponding self-energies are

$$
\begin{aligned}
& \Sigma_{N}=\sigma_{N N}^{\pi}+\sigma_{N \Delta}^{\pi} \\
& \Sigma_{\Delta}=\sigma_{\Delta \Delta}^{\pi}+\sigma_{\Delta N}^{\pi},
\end{aligned}
$$

6 Note that the $S U(3)_{c}$ colour symmetry is broken in the $2 \mathrm{SC}$ phase-the pairing is between just two colours and the third colour remains unpaired. We do not assume axial vector diquark condensation in QM, as this would break further symmetries.

7 It is possible to describe the nucleon with just the scalar diquark channel and this has been considered elsewhere [35]. As in [36], we use the approximate 'constant + pole' forms of the diquark $t$-matrices. 
where

$$
\sigma_{B B^{\prime}}^{\pi}=\frac{-3 g_{A}^{2}}{16 \pi^{2} f_{\pi}^{2}} c_{B B^{\prime}} \int_{0}^{\infty} \mathrm{d} k \frac{k^{4} u^{2}(k)}{\omega(k)\left[\omega_{B B^{\prime}}+\omega(k)\right]},
$$

where $\omega_{B B^{\prime}}=\left(M_{B}-M_{B^{\prime}}\right)$ is the physical baryon mass splitting (e.g. $\omega_{N \Delta}=1232-$ $939 \mathrm{MeV})$, and $\omega(k)=\sqrt{k^{2}+m_{\pi}^{2}}$ is the intermediate pion energy. For the $\pi B B^{\prime}$ vertex, we assume the phenomenological dipole form ${ }^{8} u(k)=\Lambda^{4} /\left(\Lambda^{2}+k^{2}\right)^{2}$. The coefficients $c_{B B^{\prime}}$ come from the standard $S U(6)$ couplings (i.e. $c_{N N}=1, c_{N \Delta}=32 / 25$ [39]). The baryon masses including the pion loop contributions are then given by $M_{B}=M_{B}^{(0)}+\Sigma_{B}$, where $M_{B}^{(0)}$ are the 'bare' masses which follow from the quark-diquark equation.

The size of the self-energy of the nucleon, $\Sigma_{N}$, is not precisely known. Calculations indicate it could be up to $-400 \mathrm{MeV}$ [39-42]. In the present work $\Sigma_{N}$ is varied by changing the dipole cut-off, $\Lambda$, within physically acceptable limits, in order to investigate how the pion cloud of the nucleon influences the equation of state of the system.

The form of the effective potential in the mean field approximation has been derived for symmetric NM in [33], starting from the quark Lagrangian, equation (1.2d), and using the hadronization method. For the present calculations the effective potential is extended to the isospin asymmetric case, because for neutron star matter charge neutrality and chemical equilibrium typically lead to an abundance of neutrons over protons. The effective potential can be written as follows:

$$
V^{\mathrm{NM}}=V_{\mathrm{vac}}+V_{N}-\frac{\omega_{0}^{2}}{4 G_{\omega}}-\frac{\rho_{0}^{2}}{4 G_{\rho}}-\frac{\mu_{e}^{4}}{12 \pi^{2}}
$$

where

$$
V_{\mathrm{vac}}=12 \mathrm{i} \int_{\mathrm{PT}} \frac{\mathrm{d}^{4} k}{(2 \pi)^{4}} \ln \frac{k^{2}-M^{2}}{k^{2}-M_{0}^{2}}+\frac{(M-m)^{2}}{4 G_{\pi}}-\frac{\left(M_{0}-m\right)^{2}}{4 G_{\pi}}
$$

is the vacuum term. The Fermi motion of the nucleons moving in the scalar and vector mean fields gives rise to the term

$V_{N}=-2 \sum_{\alpha=\mathrm{p}, \mathrm{n}} \int \frac{\mathrm{d}^{3} k}{(2 \pi)^{3}} \Theta\left(k_{F_{\alpha}}-k\right)\left(\sqrt{k_{F_{\alpha}}^{2}+M_{N}^{2}(M)}-\sqrt{k^{2}+M_{N}^{2}(M)}\right)$,

where $M_{N}(M)$ is the nucleon mass in medium, which is the sum of the bare mass and the pion cloud contribution. The relations between the chemical potentials, which are the variables of $V^{\mathrm{NM}}$, and the Fermi momenta appearing in equation (2.8) are given by

$$
\begin{aligned}
& \mu_{n}=\sqrt{M_{N}^{2}+k_{F_{n}}^{2}}+3 \omega_{0}-\rho_{0}, \\
& \mu_{p}=\sqrt{M_{N}^{2}+k_{F_{p}}^{2}}+3 \omega_{0}+\rho_{0} .
\end{aligned}
$$

The constituent quark mass, $M$, and the mean vector fields $\left(\omega_{0}\right.$ and $\left.\rho_{0}\right)$ in NM are determined by minimizing the effective potential for fixed chemical potentials ${ }^{9}$. The chemical potential of the (massless) electron in the last term of (2.6) is fixed by the requirement of beta equilibrium as $\mu_{e}=\mu_{n}-\mu_{p}$.

${ }^{8}$ The dipole cut-off $\Lambda$ should not be confused with $\Lambda_{\text {IR }}$ and $\Lambda_{\text {UV }}$ which are the cut-off parameters on the quark level in this model.

9 In the actual calculation, it is easier to minimize the energy density $\mathcal{E}=V+\sum \mu_{\alpha} \rho_{\alpha}$ for fixed densities, after eliminating the mean vector fields as $\omega_{0}=6 G_{\omega}\left(\rho_{p}+\rho_{n}\right), \rho_{0}=2 G_{\rho}\left(\rho_{p}-\rho_{n}\right)$. 
Table 1. Parameters corresponding to different choices of $\Sigma_{N}$. We define $r_{\alpha}=G_{\alpha} / G_{\pi}$ $(\alpha=a, s, \omega, \rho) . \Lambda, \Sigma_{N}$ and $\Sigma_{\Delta}$ are in MeV.

\begin{tabular}{lcccccc}
\hline$\Lambda$ & $\Sigma_{N}$ & $\Sigma_{\Delta}$ & $r_{a}$ & $r_{s}$ & $r_{\omega}$ & $r_{\rho}$ \\
\hline- & 0 & 0 & 0.27 & 0.30 & 0.48 & 0.70 \\
710 & -200 & -183 & 0.21 & 0.27 & 0.30 & 0.74 \\
803 & -300 & -266 & 0.18 & 0.24 & 0.24 & 0.76 \\
877 & -400 & -346 & 0.15 & 0.22 & 0.19 & 0.77 \\
\hline
\end{tabular}

For discussion of the phase structure of this model in section 5, we introduce the chemical potentials associated with baryon number and isospin:

$$
\mu_{B}=\frac{1}{2}\left(\mu_{p}+\mu_{n}\right), \quad \mu_{I}=\frac{1}{2}\left(\mu_{p}-\mu_{n}\right) .
$$

The parameters of the model are determined as follows. We choose $\Lambda_{\mathrm{IR}}=285 \mathrm{MeV}$ to be of the order of $\Lambda_{\mathrm{QCD}}$. We calculate $\Lambda_{\mathrm{UV}}, m$ and $G_{\pi}$ so as to reproduce $f_{\pi}=93 \mathrm{MeV}$ (through the matrix element for pion decay), $m_{\pi}=140 \mathrm{MeV}$ (through the Bethe-Salpeter equation for the pion) and constituent quark mass at zero density, $M_{0}=400 \mathrm{MeV}$ (via the gap equation). The resulting equation of state is not very sensitive to the initial choices of $\Lambda_{\mathrm{IR}}$ and $M_{0}$. The parameter $G_{\omega}$ is fixed to give the empirical binding energy per nucleon of symmetric $\mathrm{NM}\left(E_{B}=17 \mathrm{MeV}\right)$, and the parameter $G_{\rho}$ is adjusted to the symmetry energy $\left(a_{4}=32 \mathrm{MeV}\right.$ at $\rho_{0}=0.17 \mathrm{fm}^{-3}$ ). The coupling in the axial vector diquark channel, $G_{a}$, is fixed by the mass of the Delta, since in this case the scalar diquark channel does not contribute. Then, the coupling in the scalar diquark channel, $G_{s}$, is in turn determined by the nucleon mass. For the static parameter in the quark exchange kernel, we use $c=450 \mathrm{MeV}$. This calculation of baryon masses is carried out for several initial choices of the dipole cut-off $\Lambda$, which controls the magnitude of the pion loop contributions to the nucleon mass, $\Sigma_{N}$. We will show the results for three different values of $\Lambda$, leading to $\Sigma_{N}=-200 \mathrm{MeV},-300 \mathrm{MeV}$ and $-400 \mathrm{MeV}$. The resulting parameter sets are shown in table 1. (The first set corresponds to the case where the nucleon and delta masses are reproduced without pion cloud contributions.)

The function $M_{N}(M)$, which is needed in (2.8) to minimize the effective potential for finite density, is calculated by assuming that the ratio $g_{A} / f_{\pi}$ in (2.5) is independent of density. This is supported by a recent analysis of the pion-nucleus optical potential [43], which has shown that the pion decay constant in nuclear matter is reduced by $20 \%$, which is the same as the quenching of $g_{A}$ derived from Gamow-Teller matrix elements [44]. The pion mass in the medium is constrained by chiral symmetry which leads to the relation ${ }^{10} m_{\pi}(\rho)^{2}=m_{\pi}^{2} \cdot M_{0} / M$. This small enhancement of the pion mass in the medium is taken into account in the calculation but its effect is not very important. The relationship between the nucleon mass and the constituent quark mass for the four cases of table 1 is shown in figure 1 . Note that in the region $M>$ $200 \mathrm{MeV}$, which is most relevant for normal densities, both the slopes and the curvatures of the lines decrease in magnitude as the attraction from the pion loop increases. Thus, the inclusion of pion loop effects on the nucleon mass leads to a reduced effective $\sigma N N$ coupling and to a reduced scalar polarizability in the medium [1]. The self-consistently calculated quark and nucleon masses are shown as functions of the density in figure 2 .

Concerning the pion exchange effects on the NM equation of state, we note that in addition to the term which can be incorporated into the nucleon mass there is also the familiar 'Fock term' which originates from the Pauli principle. However, this contribution is quite small

\footnotetext{
${ }^{10}$ For the derivation see [1]. The pion mass in this relation is defined at zero momentum.
} 


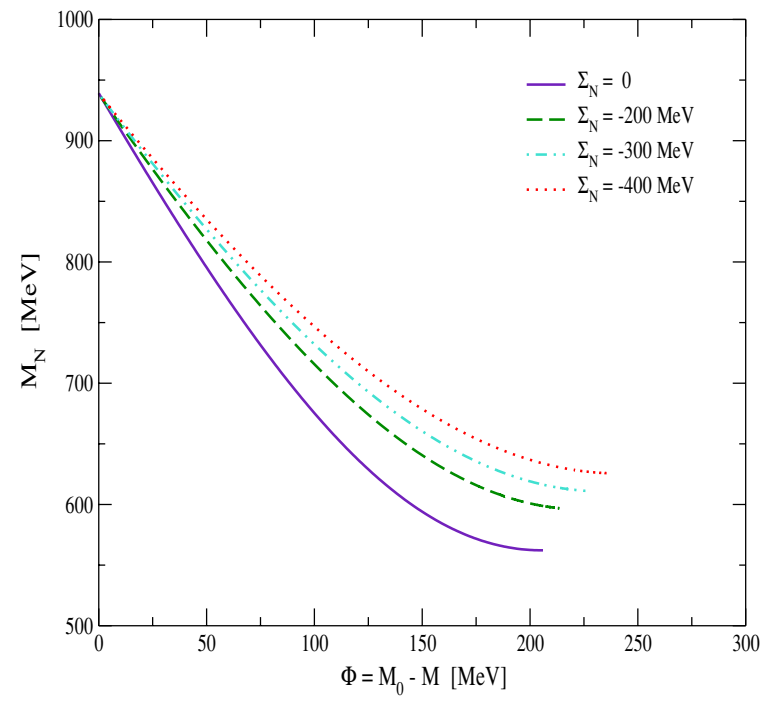

Figure 1. The mass of the nucleon as a function of the scalar potential $\left(\Phi=M_{0}-M\right)$.

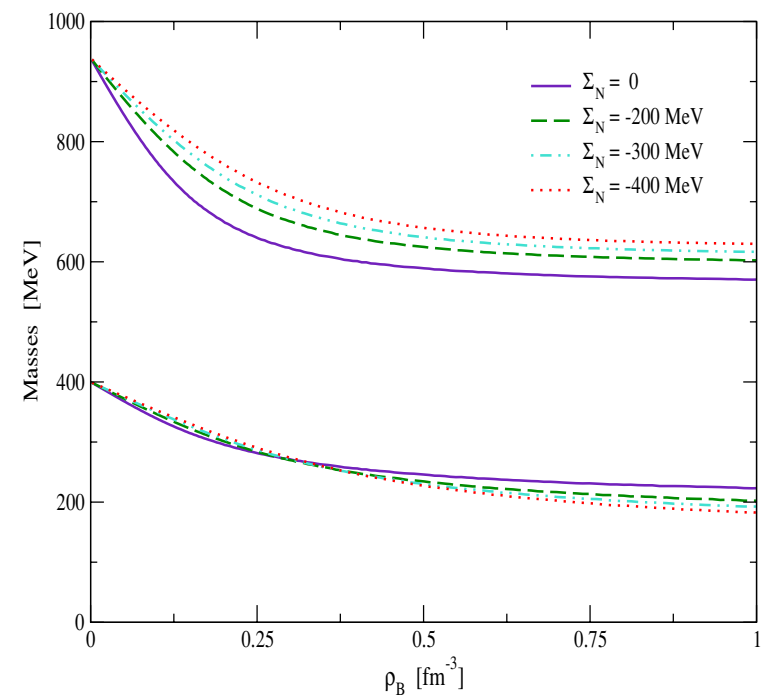

Figure 2. The effective masses of quarks (lower lines) and nucleons (upper lines) in the hadronic phase for several choices of the nucleon self-energy.

when the short-range correlations between nucleons in the spin-isospin channel are included [45], and will be neglected here for simplicity.

\section{Quark matter}

In the most simple mean field description of quark matter, each energy level for the quarks is filled up to the Fermi energy. At the Fermi surface, only a small attraction between quarks 
leads to the formation of Cooper pairs, analogous to the phenomenon of BCS pairing of electrons in a superconductor. In QCD we anticipate that colour superconductivity will arise through the $\overline{3}_{c}$ channel, reducing the energy of the system through the condensation of colour anti-symmetric pairs. The effective potential for QM thus has the form

$$
V^{\mathrm{QM}}=V_{\mathrm{vac}}+V_{Q}+V_{\Delta}-\frac{\mu_{e}^{4}}{12 \pi^{2}}
$$

where the vacuum part, $V_{\mathrm{vac}}$, is the same as for NM (equation (2.7)) except that the infrared cut-off is zero in QM. Furthermore,

$$
V_{Q}=-6 \sum_{\alpha=\mathrm{u}, \mathrm{d}} \int \frac{\mathrm{d}^{3} k}{(2 \pi)^{3}} \Theta\left(\mu_{\alpha}-E_{Q}(k)\right)\left(\mu_{\alpha}-E_{Q}(k)\right)
$$

describes the Fermi motion of quarks with chemical potentials $\mu_{u}$ and $\mu_{d}$, and $E_{Q}(k)=$ $\sqrt{M^{2}+k^{2}}$. The term (3.2) is analogous to $V_{N}$ in NM, except that the quark mass, $M$, corresponds directly to the scalar field in the system, i.e., there is no scalar polarizability of the quarks. The term $V_{\Delta}$ describes the effect of the pairing gap and is given by

$V_{\Delta}=2 \mathrm{i} \int_{\mathrm{PT}} \frac{\mathrm{d}^{4} k}{(2 \pi)^{4}} \sum_{\alpha=+,-}\left[\ln \frac{k_{0}^{2}-\left(\epsilon_{\alpha}+\mu_{I}\right)^{2}}{k_{0}^{2}-\left(E_{\alpha}+\mu_{I}\right)^{2}}+\ln \frac{k_{0}^{2}-\left(\epsilon_{\alpha}-\mu_{I}\right)^{2}}{k_{0}^{2}-\left(E_{\alpha}-\mu_{I}\right)^{2}}\right]+\frac{\Delta^{2}}{6 G_{s}}$,

where $\epsilon_{ \pm}(k)=\sqrt{\left(E_{Q}(k) \pm \mu_{B} / 3\right)^{2}+\Delta^{2}}, E_{ \pm}=\left|E_{Q}(k) \pm \mu_{B} / 3\right|$, and we introduced the chemical potentials for baryon number and isospin ${ }^{11}$

$$
\mu_{B}=\frac{3}{2}\left(\mu_{u}+\mu_{d}\right), \quad \mu_{I}=\frac{1}{2}\left(\mu_{u}-\mu_{d}\right),
$$

which corresponds to (2.11) in the NM phase. The electron chemical potential is determined from beta equilibrium as $\mu_{e}=\mu_{d}-\mu_{u}=-2 \mu_{I}$.

The gap $\Delta$ and the quark mass $M$ are determined by minimizing the effective potential for fixed chemical potentials. Our results, discussed below, show that $M$ is quite small in the QM phase, i.e., we have almost current quarks.

Note that the vector-type interactions are set to zero in QM, even though in the description of NM the vector mean fields are clearly important. This assumption, which has been made implicitly in almost all investigations of high density quark matter, is supported by the discussions of vector meson poles in [33]. It is also supported by recent arguments related to the EMC effect [48], which show that in the high energy region, where one has essentially current quarks (as in the present high density case), the mean vector field must indeed be set to zero.

In the following discussions, we distinguish the normal quark matter (NQM) phase, which is characterized by $\Delta=0$, from the colour superconducting quark matter (SQM) phase $(\Delta>0)$. Note that the value of $G_{s}=r_{s} G_{\pi}$ controls the outcome of this minimization through the last term in (3.3). In these calculations, we focus on the case where the pairing strength $\left(r_{s}\right)$ in QM takes the same value as in NM, namely the value required to obtain the correct nucleon mass after the pion cloud contribution is taken into account.

\section{Phase diagrams and mixed phases}

To construct a phase diagram in the plane of the chemical potentials, as in figure 3 which is discussed below, we compare the effective potentials for the NM, NQM and SQM phases at

\footnotetext{
${ }^{11}$ We mention that in principle one needs a further chemical potential for colour neutrality $\left(\mu_{8}\right)$ in QM. However, for
} the two-flavour case $\mu_{8}$ turns out to be very small [15, 46, 47]. 

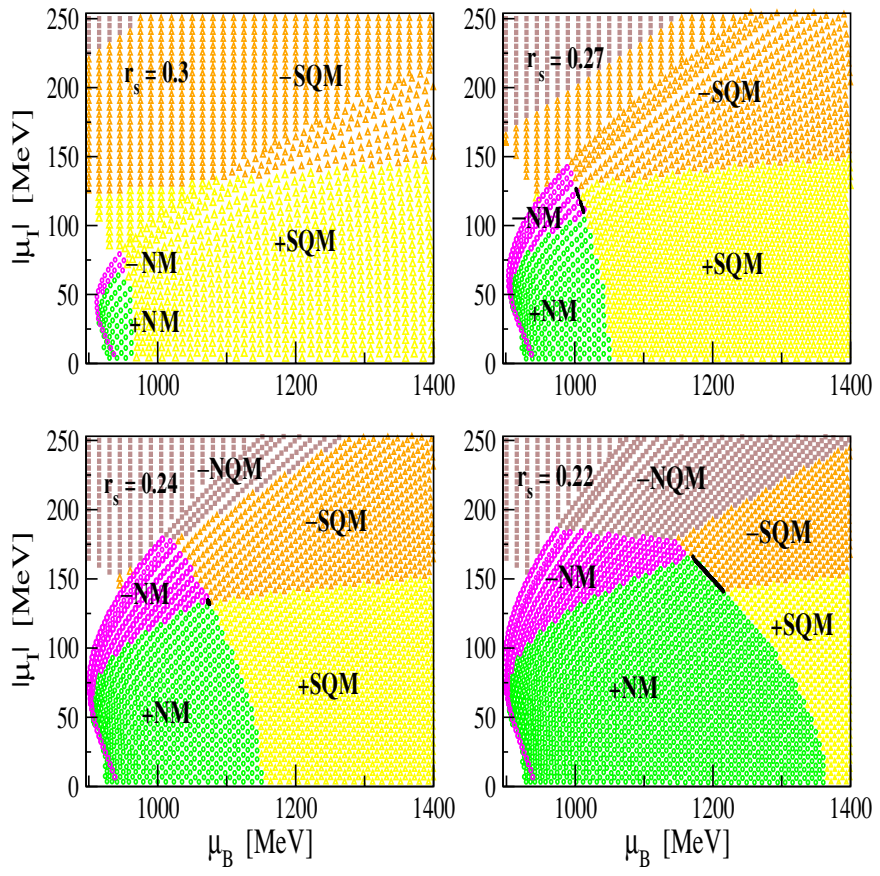

Figure 3. Phase diagrams with $r_{s}(\mathrm{NM})=r_{s}(\mathrm{QM})$. The nucleon self-energies $\left(\Sigma_{N}\right)$ are 0 , $-200,-300$ and $-400 \mathrm{MeV}$, respectively. In each region, the sign of the charge density is indicated. Charge neutral matter occurs at the boundary of the positively and negatively charged regions. The mixed phases correspond to the black lines.

each point. The phase with the smallest effective potential (largest pressure) is the one which is physically realized. At each point, we calculate the baryon and charge densities

$$
\begin{aligned}
& \rho_{B}^{a}=-\frac{\partial V^{a}}{\partial \mu_{B}}, \\
& \rho_{c}^{a}=-\frac{1}{2}\left(\frac{\partial V^{a}}{\partial \mu_{B}}+\frac{\partial V^{a}}{\partial \mu_{I}}\right),
\end{aligned}
$$

where $a=\mathrm{NM}$, NQM or SQM. The actual equation of state as a function of baryon density is then constructed according to global charge neutrality. If charge neutrality can be realized within one phase (for example the NM phase), one simply moves along the charge neutral line in the phase diagram (for example, the line $+\mathrm{NM} /-N M$ in figure 3 ) as the baryon density increases. When a phase transition occurs, it is necessary to construct a mixed phase, which is composed of positively and negatively charged components belonging to two different phases [49]. A charge neutral mixture of NM and QM (where QM refers to either NQM or SQM), for example, is characterized by the volume fraction,

$$
\chi^{\mathrm{NM}}=\frac{\rho_{c}^{\mathrm{QM}}}{\rho_{c}^{\mathrm{QM}}-\rho_{c}^{\mathrm{NM}}},
$$

which ranges from 0 to 1 , as the density increases from the point of pure $\mathrm{NM}$ (where $\rho_{c}^{\mathrm{NM}}=0$ ) to the one of pure QM (where $\rho_{c}^{\mathrm{QM}}=0$ ). 
The baryon and energy densities for the mixed phase are then expressed by the volume fraction as follows:

$$
\begin{aligned}
& \rho_{B}^{M}=\chi^{\mathrm{NM}} \rho_{B}^{\mathrm{NM}}+\left(1-\chi^{\mathrm{NM}}\right) \rho_{B}^{\mathrm{QM}}, \\
& \mathcal{E}^{M}=\chi^{\mathrm{NM}} \mathcal{E}^{\mathrm{NM}}+\left(1-\chi^{\mathrm{NM}}\right) \mathcal{E}^{\mathrm{QM}} .
\end{aligned}
$$

Note that the components of the mixed phase have equal pressures $\left(P^{M}=P^{\mathrm{NM}}=P^{\mathrm{QM}}\right)$ at each point $\left(\mu_{B}, \mu_{I}\right)$ on the phase boundary. In this way, one moves along the phase boundaries between NM and QM while $\rho_{B}$ is increasing, until one comes to the point where charge neutral pure $\mathrm{QM}$ is realized $\left(\chi^{\mathrm{NM}}=0\right)$

In practice, our procedure is as follows. We first find the point where the effective potential for charge neutral NM becomes equal to the one for QM. At this point $\chi^{\mathrm{NM}}=1$, since $\rho_{c}^{\mathrm{NM}}=0$. From this point, we incrementally increase either the neutron or proton density (depending on whether the transition is in the direction of increasing neutron and/or proton density). For example, the transition from NM to SQM $\left(r_{s}=0.27\right)$ is in the direction of increasing neutron density. For each neutron density, we determine the value for proton density required to ensure that we are on the phase boundary. Next, we calculate the charge densities of each phase and the resulting volume fraction. The volume averaged properties of the mixed phase are then related to its component phases by equations (4.4) and (4.5). This process continues until we encounter the point where the QM phase becomes charge neutral $\left(\rho_{c}^{\mathrm{QM}}=0\right)$, and thus the volume fraction $\chi^{\mathrm{NM}}$ goes to 0 . From here, the remainder of the equation of state will be the pure charge neutral QM phase.

\section{Results}

The equations of state for this model exhibit phase transitions from the confined quark-diquark states employed in the description of NM to a phase with condensed quark pairs in the form of colour superconducting QM. The key point in our present work is to equate the pairing strength for the colour superconducting pairs in QM with the scalar diquark interactions inside the nucleon. Depending on the amount of attractive contributions of the pion cloud to the nucleon mass, this gives rise to a series of phase diagrams shown in figure 3, corresponding to the four cases of table 1 .

The four diagrams in figure 3 are shown for increasing pion cloud contributions to the nucleon mass, i.e., decreasing scalar diquark interactions characterized by the ratio $r_{s}$. Starting from the first diagram in figure 3, the regions occupied by SQM become smaller while those of NM and NQM phases become larger. In the first phase diagram, where the pion cloud is not included and the whole attraction within the nucleon is attributed to the diquark correlations, the NM phase is expelled almost completely and overwhelmed by the SQM phase even in the low density region. Since this is clearly unphysical, we can conclude that the naive quarkdiquark picture of the nucleon leads to a phase structure which is in conflict with empirical evidence. Hence, some attraction within the nucleon must be attributed to the pion cloud.

The second diagram in figure 3, which corresponds to a mass shift of $\Sigma_{N}=-200 \mathrm{MeV}$ from the pion cloud, involves a mixed phase $-\mathrm{NM} /+\mathrm{SQM}$ before the system undergoes a transition to the pure SQM phase. The phase boundary in this case is rather short, indicating that the pressure is almost constant during the phase transition. In the third diagram $\left(\Sigma_{N}=\right.$ $-300 \mathrm{MeV}$ ), the NM region extends to larger values of $\mu_{B}$, and the charge neutral phase boundary shrinks almost to a point. The fourth diagram $\left(\Sigma_{N}=-400 \mathrm{MeV}\right)$ involves a mixed phase $+\mathrm{NM} /-\mathrm{SQM}$ before the pure SQM phase is reached. 

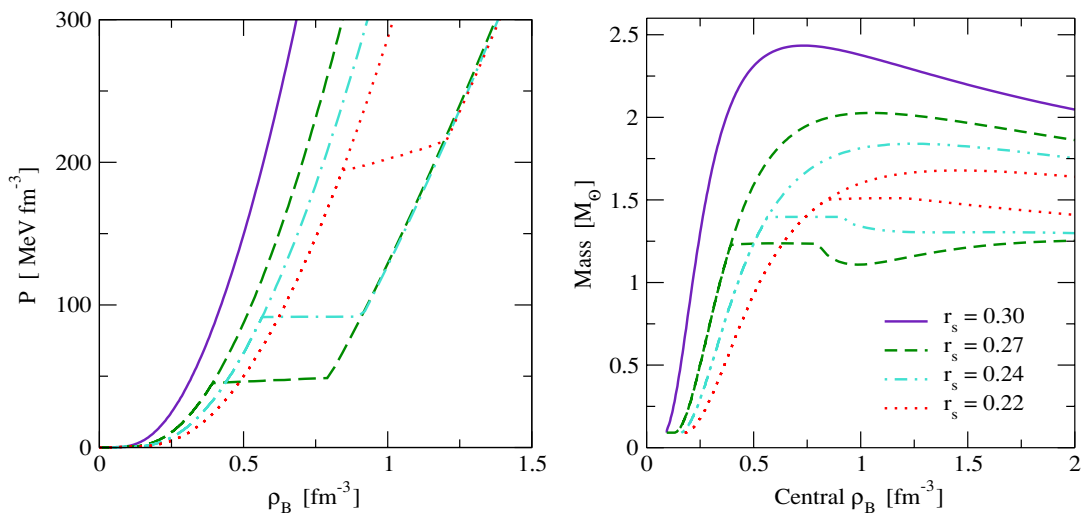

Figure 4. Equations of state and neutron star masses for the choices of scalar attraction described in the text.

The charge neutral equations of state for these four cases are shown on the left-hand side of figure 4. In the low density region $\left(0>\rho_{B}>0.001 \mathrm{fm}^{-3}\right)$, we use the equation of state of Negele and Vautherin [50] to describe the neutron star crust. On the NM side of figure 4, the equation of state becomes softer with increasing pion cloud contributions, $\Sigma_{N}$. This can be understood from our discussions in section 2. In particular, the inclusion of the pion cloud leads to a reduction of the effective $\sigma N N$ coupling in the medium, which must be balanced by a smaller vector coupling (the parameter $r_{\omega}$ in table 1) in order to maintain the correct binding energy at the nuclear matter density $0.17 \mathrm{fm}^{-3}$. Note that the actual saturation point for the model moves to somewhat higher densities $\left(0.14,0.19,0.21\right.$ and $\left.0.25 \mathrm{fm}^{-3}\right)$ when $\Sigma_{N}$ increases $(0,-200,-300$ and $-400 \mathrm{MeV}$, respectively). As noted in earlier works, the only parameter in the present model that may be adjusted to give the saturation point is $G_{\omega}$ [29]. With this in mind, these results are reasonably close to the empirical value of $\rho_{0}=0.17 \mathrm{fm}^{-3}$.

On the QM side all cases considered here give almost identical equations of state, indicating that the relationship between density and pressure in charge neutral SQM is not sensitive to the value of $r_{s}$ (or indeed to the value of $\Delta$ ). However, the transition densities are sensitive to $r_{s}$. In all cases considered here, the pressure variations in the mixed phase are rather small, indicating that our construction, based on the Gibbs criteria of phase equilibrium, gives similar results to a naive Maxwell construction between charge neutral NM and SQM.

Through the Tolman-Oppenheimer-Volkoff (TOV) equations [51], any equation of state specifies a unique set of non-rotating relativistic stars. The right-hand side of figure 4 illustrates the solutions to these equations for each of the equations of state on the left-hand side of figure 4 . We see from these figures that the inclusion of pion cloud contributions to the nucleon mass, leading to a decreasing strength of scalar diquark pairing, has significant effects on the properties of neutron stars. Because of the softening of the equation of state, the maximum masses for pure hadronic stars are reduced from 2.4 solar masses to 2.0, 1.8 and 1.7 solar masses as $\Sigma_{N}$ increases (and $r_{s}$ decreases accordingly).

The equations of state with phase transitions to SQM produce plateaus in the central density versus mass curves in figure 4 . In the case of $r_{s}=0.22$, only the mixed phase can be reached inside a star, since a region of negative slope in this plot corresponds to unstable solutions to the TOV equation. However, stable hybrid stars with quark cores are possible in the case of $r_{s}=0.27$ and $r_{s}=0.24$. In these configurations there are additional sets of stable solutions at higher densities, which in the literature are referred to as twins [52] (since 


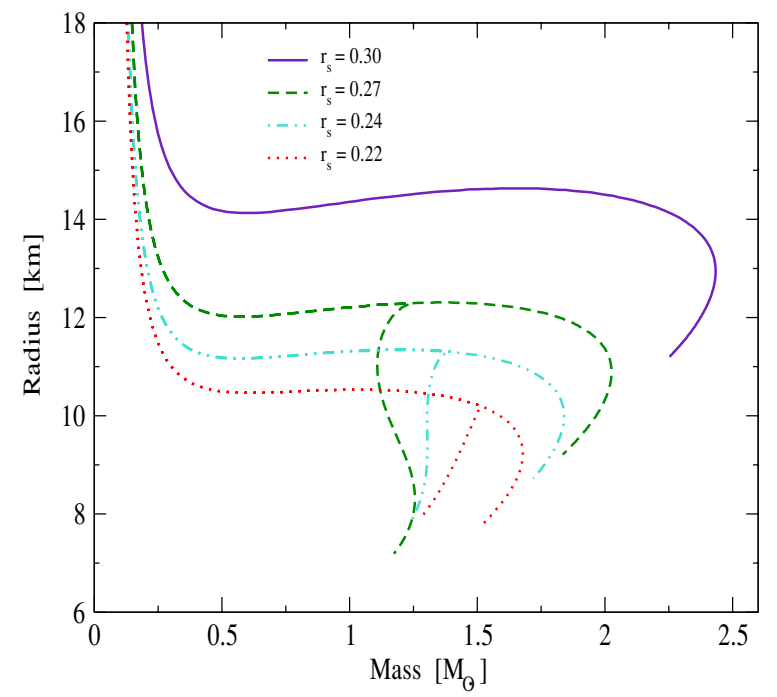

Figure 5. Mass-radius relationship of neutron stars and hybrid stars for the choices of scalar attraction described in the text.

for these configurations there can be stars that have the same masses but different radii, as illustrated in figure 5). Qualitatively, similar results are also found in [53]. It has recently been shown that the second set of solutions is indeed stable and may give rise to an observable signature for the occurrence of phase transitions in compact stars [54].

However, in the case of $r_{s}=0.27$, the masses are too small to allow for observed pulsar masses, which are typically about 1.4 solar masses. For $r_{s}=0.24$ and $r_{s}=0.22$, the maximum masses are approximately 1.4 and 1.5 solar masses, respectively. One possibility that may give rise to more massive hybrid stars in this model is to delay the phase transition to QM. If hyperons were included, for example, then the transition may be shifted to higher densities, since the equation of state in the hadronic phase would be softened independent of the value of the pairing strength $r_{s}$. We note that the correlation between the transition densities and the maximum neutron star masses shown in figure 4 follows the phenomenological discussions on phase transitions given in [56].

It is interesting to note that these phase transitions to QM give rise to plateaus in the neutron star masses. This phenomenon may be the reason that so many observed neutron stars lie within such a narrow mass range [55]. In addition, figure 5 shows that the radii of the stars may be reduced by $2-4 \mathrm{~km}$ if the pion cloud contribution to the nucleon mass increases (and the scalar pairing interaction decreases accordingly).

\section{Discussion}

We have used the NJL model, supplemented with a method of regularization which simulates confinement, to calculate consistently the properties of nuclear matter and quark matter. In doing so, we have related the quark interactions in the confined phase, to the quark interactions in the deconfined phase, where colour superconductivity is assumed to arise. Our principal finding is that the scalar pairing between quarks within the nucleon and in QM may be equated, if the attraction within the nucleon is attributed not only to the diquark interactions but also to 
the pion cloud. Since the attraction in the scalar $\overline{3}_{c}$ channel between (almost) current quarks at high densities or energies can be derived directly from QCD, this result lends some support to the Faddeev approach to the nucleon, since the quark-diquark picture may be characterized by not only the same type of pairing interaction but also the same strength as we expect to find in the high density QM phase. This is an important feature of nucleon dynamics and is relevant to any finite density studies that incorporate nucleon structure.

By including the pion cloud contributions to the nucleon mass, we found that the equation of state of nuclear matter becomes softer, reducing the neutron star and hybrid star masses significantly.

\section{Acknowledgments}

SL would like to thank Ian Cloet for helpful discussions. This work was supported by the Australian Research Council and DOE contract DE-AC05-84ER40150, under which SURA operates Jefferson Lab, and by the Grant in Aid for Scientific Research of the Japanese Ministry of Education, Culture, Sports, Science and Technology, project no C2-16540267.

\section{References}

[1] Bentz W and Thomas A W 2001 Nucl. Phys. A 696138

[2] Guichon P A M 1988 Phys. Lett. B 200235

Guichon P A M and Thomas A W 2004 Phys. Rev. Lett. 93132502

[3] Rezaeian A H and Pirner H 2005 Preprint nucl-th/0510041

[4] Thomas A W et al 2004 Prog. Theor. Phys. Suppl. 156 124-36

[5] Serot B D and Walecka J D 1986 Adv. Nucl. Phys. 111

[6] Wilczek F 2004 Preprint hep-ph/0409168

[7] Santopinto E 2005 Phys. Rev. C 72 022201(R)

[8] Capstick S and Isgur N 1986 Phys. Rev. D 342809

[9] Lugones G and Bombaci I 2005 Phys. Rev. D 72065021

Rischke D H 2004 Prog. Part. Nucl. Phys. 52197

Schäfer T and Wilczek F 1999 Phys. Rev. D 60114033

Grigorian H, Blaschke D and Aguilera D N 2004 Phys. Rev. C 69065802

[10] Bandyopadhyay D 2005 Preprint nucl-th/0512100

[11] Alford M 2005 Preprint nucl-th/0512005

[12] Weber F 2005 Prog. Part. Nucl. Phys. 54193 and references therein

[13] Wang P et al 2005 Phys. Rev. C 72045801

[14] Itoh N 1970 Prog. Theor. Phys. 44291

[15] Buballa M 2005 Phys. Rep. 407205

[16] Blaschke D et al 2005 Phys. Rev. D 72065020

[17] Rüster S, Werth V, Buballa M, Shovkovy I A and Rischke D H 2005 Phys. Rev. D 72034004

[18] Buballa M et al 2004 Phys. Lett. B 595 36-43

[19] He L y, Jin M and Zhuang P f 2005 Phys. Rev. D 71116001

[20] Barducci A, Casalbuoni R, Pettini G and Ravagli L 2005 Phys. Rev. D 71016011

[21] Ebert D and Klimenko K G 2005 Preprint hep-ph/0507007

[22] Ebert D and Klimenko K G 2005 Preprint hep-ph/0510222

[23] Maruyama T et al 2005 Nucl. Phys. A $760319-45$

[24] Warringa H J, Boer D and Andersen J O 2005 Phys. Rev. D 72014015

[25] Nambu Y and Jona-Lasinio G 1960 Phys. Rev. 122345

Nambu Y and Jona-Lasinio G 1961 Phys. Rev. 124246

see also Vogl U and Weise W 1991 Prog. Part. Nucl. Phys. 27195

[26] Buballa M 1996 Nucl. Phys. A 611 393-408

[27] Koch V et al 1987 Phys. Lett. B 1851

[28] da Providencia J et al 1987 Phys. Rev. D 361882

[29] Ishii N, Bentz W and Yazaki K 1995 Nucl. Phys. A 578617 
[30] Klevansky S P 1992 Rev. Mod. Phys. 64649

[31] Ebert D, Feldmann T and Reinhardt H 1996 Phys. Lett. B 388154

[32] Hellstern G, Oettel M, Alkofer R and Reinhardt H 1998 Preprint hhep-ph/9805393

[33] Bentz W, Horikawa T, Ishii N and Thomas A W 2003 Nucl. Phys. A 72095

[34] Buck A, Alkofer R and Reinhardt H 1992 Phys. Lett. B 28629

[35] Lawley S, Bentz W and Thomas A W 2006 Phys. Lett. B 632495

[36] Cloet I, Bentz W and Thomas A W 2005 Phys. Lett. B 621246

[37] Schwinger J S 1951 Phys. Rev. 82664

[38] Thomas A W and Weise W 2001 The Structure of the Nucleon (Berlin: Wiley-VCH)

[39] Young R D et al 2002 Phys. Rev. D 66094507

[40] Thomas A W, Théberge S and Miller G A 1981 Phys. Rev. D 24216

[41] Pearce B C and Afnan I R 1986 Phys. Rev. C 34991

[42] Hecht M et al 2002 Phys. Rev. C 65055204

[43] Suzuki K et al 2004 Phys. Rev. Lett. 92072302

[44] Arima A, Shimizu K, Bentz W and Hyuga H 1987 Adv. Nucl. Phys. 181

[45] Ericson T and Weise W 1988 Pions and Nuclei (Oxford: Clarendon) p 137

[46] Grigorian H, Blaschke D and Aguilera D N 2004 Phys. Rev. C 69065802

[47] Aguilera D N, Blaschke D and Grigorian H 2005 Nucl. Phys. A 757527

[48] Detmold W, Miller G A and Smith J R 2005 Preprint nucl-th/0509033

[49] Glendenning N K 1992 Phys. Rev. D 461274

[50] Negele J and Vautherin D 1973 Nucl. Phys. A 207298

[51] Oppenheimer J R and Volkoff G M 1939 Phys. Rev. 55374

[52] Glendenning N K and Kettner C 2000 Astron. Astrophys. 353 L9

[53] Fraga E S, Pisarski R D and Schaffner-Bielich J 2001 Phys. Rev. D 63121702

[54] Schertler K, Greiner C, Schaffner-Bielich J and Thoma M H 2000 Nucl. Phys. A $677463-90$

[55] Lattimer J M and Prakash M 2005 Phys. Rev. Lett. 94111101

[56] Takahara M and Sato K 1988 Astrophys. J. 335301 